\title{
Incentive-Elicited Brain Activation in Adolescents: Similarities and Differences from Young Adults
}

\author{
James M. Bjork, ${ }^{1}$ Brian Knutson, ${ }^{2}$ Grace W. Fong, ${ }^{1}$ Daniel M. Caggiano, ${ }^{3}$ Shannon M. Bennett, ${ }^{1}$ and Daniel W. Hommer ${ }^{1}$ \\ ${ }^{1}$ Laboratory of Clinical Studies, National Institute on Alcohol Abuse and Alcoholism-National Institutes of Health, Bethesda, Maryland 20892-7003, \\ ${ }^{2}$ Psychology Department, Stanford University, Stanford, California 94305, and ${ }^{3}$ Psychology Department, The Catholic University of America, Washington, \\ DC 20064
}

\begin{abstract}
Brain motivational circuitry in human adolescence is poorly characterized. One theory holds that risky behavior in adolescence results in part from a relatively overactive ventral striatal (VS) motivational circuit that readily energizes approach toward salient appetitive cues. However, other evidence fosters a theory that this circuit is developmentally underactive, in which adolescents approach more robust incentives (such as risk taking or drug experimentation) to recruit this circuitry. To help resolve this, we compared brain activation in 12 adolescents (12-17 years of age) and 12 young adults (22-28 years of age) while they anticipated the opportunity to respond to obtain monetary gains as well as to avoid monetary losses. In both age groups, anticipation of potential gain activated portions of the VS, right insula, dorsal thalamus, and dorsal midbrain, where the magnitude of VS activation was sensitive to gain amount. Notification of gain outcomes (in contrast with missed gains) activated the mesial frontal cortex (mFC). Across all subjects, signal increase in the right nucleus accumbens during anticipation of responding for large gains independently correlated with both age and self-rated excitement about the high gain cue. In direct comparison, adolescents evidenced less recruitment of the right VS and right-extended amygdala while anticipating responding for gains (in contrast with anticipation of nongains) compared with young adults. However, brain activation after gain outcomes did not appreciably differ between age groups. These results suggest that adolescents selectively show reduced recruitment of motivational but not consummatory components of reward-directed behavior.
\end{abstract}

Key words: accumbens; activation; aging [ageing]; development; imaging; motivation; reward; adolescence

\section{Introduction}

Adolescence is characterized by a normative increase in "risky" behavior (Furby and Beyth-Marom, 1992), in which underdeveloped incentive neural circuitry may underlie both impulsivity and addiction vulnerability (Chambers et al., 2003). Compared with adults, adolescents: (1) are less apt to consider the negative repercussions of rewarded behavior in hypothetical scenarios (Tangney et al., 1996; Reppucci, 1999), (2) base decisions on temporally proximal outcomes rather than distal outcomes (Gardner and Herman, 1991), and (3) in some contexts, are better motivated by reward than by negative reinforcement (Gardner and Herman, 1991; Arnett, 1992). Here, we explored whether adolescents differ from young adults in cue-elicited activation of mesolimbic motivational circuitry.

Functional magnetic resonance imaging (FMRI) provides a noninvasive tool for visualizing neural correlates of incentive processing in humans and can measure metabolic activity in sub-

Received Sept. 24, 2003; accepted Dec. 22, 2003.

This research was sponsored by intramural research funds from the National Institute on Alcohol Abuse and Alcoholism. During manuscript preparation, J.B. was supported by a Pharmacology Research Associate Program fellowship from the National Institute of General Medical Sciences, and B.K. was supported by National Institute of Mental Health Grant MH066923 and a National Alliance for Research on Schizophrenia and Depression Young Investigator Award. We thank Reza Momenan for technical support.

Correspondence should be addressed to Dr. James M. Bjork, National Institute on Alcohol Abuse and AlcoholismNational Institutes of Health, 10 Center Drive, Room 3C-103, Bethesda, MD 20892. E-mail: jbjork@mail.nih.gov. DOI:10.1523/JNEUROSCI.4862-03.2004

Copyright $\odot 2004$ Society for Neuroscience $\quad 0270-6474 / 04 / 241793-10 \$ 15.00 / 0$ cortical regions implicated in incentive processing in animal models. Using FMRI, our laboratory has attempted to dissociate anticipatory (motivational) from outcome (consummatory) components of reward processing in healthy adults. We repeatedly found that anticipating the opportunity to respond for monetary gain activates regions of the ventral striatum (VS) (Knutson et al., 2001a), whereas notification of successful responses activated a region of the mesial frontal cortex ( $\mathrm{mFC}$ ) (Knutson et al., $2001 \mathrm{a}, \mathrm{b})$. One interpretation of this dissociation is that the VS responds to learned appetitive cues (Knutson et al., 2001a) to energize expenditure of effort (Salamone and Correa, 2002; Salamone et al., 2003) to obtain a potential reward, whereas the $\mathrm{mFC}$ directs this energy toward appropriate goal objects (Elliott et al., 2000).

Some theorists have postulated that adolescent risk-taking behavior might be driven by disproportionately increased activation of the VS motivational circuit by potential gain cues relative to the influence of inhibitory circuits (Chambers et al., 2003). However, others have argued that adolescent risk taking might result from a relative deficit in the activity of the VS, which requires intense appetitive stimuli (such as high-risk-high-reward incentives) to stimulate (Spear, 2000). Alternatively, adolescents may be especially motivated by the potential for immediate reinforcement resulting from underdeveloped neural circuitry required to maintain motivation for gain during delays between indication of potential gain and performance of the behavior 
required to obtain it. For example, maturation of the caudate is delayed in children with disorders characterized by deficiencies in delayed gratification (Castellanos et al., 2002), and rats with lesions of the nucleus accumbens (NAcc) core displayed decreased choice for larger but delayed reward (Cardinal et al., 2001). Thus, these surfeit versus deficit models of adolescent incentive processing make opposite predictions about the activity of the VS in response to incentive cues. Here, using FMRI in conjunction with a task that distinguishes between anticipation of working for monetary incentives and their outcomes, we examined whether adolescents would show either increased or reduced VS activation during anticipation of responding for monetary incentives compared with young adults.

\section{Materials and Methods}

Subjects. All procedures were reviewed and approved by the National Institute on Alcohol Abuse and Alcoholism Institutional Review Board. Twelve right-handed adolescent subjects $12-17$ years of age (six males; mean age, $14.75 \pm 1.9)$ and 12 right-handed young adult subjects $21-28$ years of age (six males; mean age, $23.8 \pm 2.0$ ) participated with written informed consent. Subjects were free of any physical or mental illness, as determined by structured clinical interviews from the Diagnostic and Statistical Manual for Mental Disorders, Fourth Edition, and a physical exam with complete blood count and clinical chemistries. To ensure that adolescent subjects were free of behavior problems, subclinical levels of behavior and mood symptomatology were assessed by both parent- and self-completed versions of the computerized child behavior checklist (CBCL) (Achenbach, 1991). To maximize the sensitivity of the CBCL, the higher rating of the parent or adolescent was used as the measure of the adolescent's symptomatology. CBCL responses indicated no incidence of either internalizing or externalizing symptomatology above the normal range in the adolescents.

Monetary incentive delay task. The monetary incentive delay (MID) task was designed to delineate brain activation specific to the anticipation of working to obtain potential monetary gain or avoid loss versus activation related to processing gain or loss outcomes (Knutson et al., 2000). It can be parametrically varied to examine the effects of incentive values (Knutson et al., 2001a). Subjects were instructed to respond with a button press on a hand-held button box while a white square target was displayed. Subjects could either win money or avoid losing money, as indicated by different cues presented before each target. MID stimuli were presented on a screen at the foot of the scanner bed by a projection monitor and viewed via mirrors mounted on the head coil. Each $6 \mathrm{sec}$ trial was contiguous (no intertrial interval) and was comprised of several events: cue, anticipation, response to target, and feedback, as follows.

First, one of seven cue shapes was presented for $250 \mathrm{msec}$. Three gain (reward) cues signaled that if the subject responded during the subsequent target presentation, he or she would win money. Amounts included 20 cents ( 18 trials; a circle with one horizontal line), 1 dollar (18 trials; a circle with two horizontal lines), or 5 dollars (18 trials; a circle with three horizontal lines). Conversely, three potential loss cues signaled a possibility of losing 20 cents (18 trials; a square with one horizontal line), 1 dollar (18 trials; a square with two horizontal lines), or 5 dollars (18 trials; a square with three horizontal lines) if the subject failed to respond to the subsequent target before it disappeared. As a control condition, cues signaling nonincentive outcomes ( 36 trials; denoted by triangles) were also presented, and subjects were instructed to respond as usual to the target during these trials; however, their success would not alter their winnings. Trial types (defined by the cue type) were pseudorandomly ordered.

Second, the cue was replaced by a cross-hair for a variable interval (anticipation period, $2000-2500 \mathrm{msec}$ ). Third, a white target square was presented for a variable length of time (180-280 msec). Fourth, the trial concluded with immediate feedback $(1650 \mathrm{msec})$ after the disappearance of the target, which notified participants whether they had won or lost money during that trial and also displayed their cumulative earnings.

Before scanning, each subject was familiarized with the task in a 5 min practice session in another testing laboratory. During this session, reaction times to gain targets, loss-avoidance targets, and nonincentive targets were covertly measured. For the actual scan session, the distribution of target presentation durations (identical for each cue type) was then individually set, such that each participant would succeed on $\sim 66 \%$ of trials. Before scanning, participants were also shown the cash they could earn inside an envelope to enhance the motivational salience of the task. Once in the scanner, each participant engaged in two 72-trial runs of the MID task during functional scan acquisition (described below). After the MID task, participants underwent a structural scan (described below) for anatomical colocalization. During this scan, we assessed individual differences in motivational salience of the cues. Participants used the button box to rate (one to four) how they felt when they saw each of the task cues on four-point Likert scales (e.g., how "happy," "tired," or "tense" each cue made them feel). Once the subject was placed, the localization and functional and structural scanning lasted $\sim 45 \mathrm{~min}$.

FMRI acquisition. Imaging was performed using a $3 \mathrm{~T}$ General Electric MRI scanner (General Electric, Milwaukee, WI) and a standard quadrature head coil. To provide higher spatial resolution of subcortical structures than previous studies using this paradigm, we collected $242.0 \mathrm{~mm}$ thick contiguous axial slices drawn from the base of the orbitofrontal cortex upward to the level approximately at the apex of the corpus callosum. This montage encompassed several regions of interest described in previous research using reward paradigms, including orbitofrontal cortex, $\mathrm{mFC}$, ventral and dorsal striatum, amygdala, insula, and thalamus. In-plane resolution was $3.75 \times 3.75 \mathrm{~mm}$. Functional scans were acquired using a $2^{*}$-sensitive echoplanar sequence with a repetition time (TR) of $2000 \mathrm{msec}$, echo time (TE) of $40 \mathrm{msec}$, and flip equal to $90^{\circ}$. To allow for signal stabilization before events of each run, six acquisitions were obtained before task onset. A total of 560 volumes was collected across both runs. Structural scans were acquired using a T1-weighted MP-RAGE (magnetization-prepared rapid gradient echo) sequence (TR, $100 \mathrm{msec}$; TE, $7 \mathrm{msec}$; flip, $90^{\circ}$ ), which facilitated localization and coregistration of functional data. To minimize head movement during task performance, each subject affixed his or her teeth to a custom-molded bite bar.

FMRI analysis. Analyses focused only on changes in blood oxygen level-dependent (BOLD) signal contrast (hereafter, activation) that occurred after anticipatory delay periods and trial feedback and were conducted using Analysis of Functional Neural Images (AFNI) software (Cox, 1996). We directly statistically compared FMRI data of adults and adolescents, because pediatric functional neuroimaging studies suggest that even small children show similar hemodynamic responses to adults in several event-related FMRI tasks (for review, see Gaillard et al., 2001).

This analysis was conducted (and is presented) in two stages. First, statistical maps were generated for each group separately by conventional time-course contrasts between events of interest with hemodynamically convolved models. Second, to directly compare groups, two subsequent analyses were performed: direct $t$ test comparison of the activation maps themselves (a comparison of normalized event-related $\beta$ weights) as well as BOLD signal changes in volumes of interest (VOI) selected $a$ priori in areas recruited in incentive processing in the MID task in previous reports.

Echoplanar image volumes were preprocessed in AFNI as follows: (1) volumes were concatenated across both task sessions, (2) voxel time series were interpolated to correct for nonsimultaneous slice acquisition within each volume (using sinc interpolation and the most inferior slice as a reference), and (3) volumes were corrected for motion in threedimensional space. The third from final volume collected during the MID task was used as the reference volume. Motion-correction estimates indicated that no participant's head moved $>1.5 \mathrm{~mm}$ in any dimension from one volume acquisition to the next. Across the entire task, no participant's head moved $>3 \mathrm{~mm}$ overall in any dimension. Visual inspection of volumes over time ("cineloop") indicated minimal residual head motion after the volume correction. We imposed a $4 \mathrm{~mm}$ full-width half-maximum (FWHM) smoothing kernel in the spatial domain to maximize the signal-to-noise ratio. Finally, a despiking algorithm was applied to the data on a voxel-wise basis to smooth out deviations in signal $>2.5$ SD from mean, followed by a bandpass filtering algorithm 
that smoothed cyclical fluctuations in signal that were not temporally indicative of a hemodynamic response (either $>0.011 / \mathrm{sec}$ or $<0.15 / \mathrm{sec}$ ).

Preprocessed time series data for each individual were then analyzed by multiple regression (Neter et al., 1996), which allowed covariation of variables related to head motion and scanning run. The regression model consisted of four orthogonal regressors of interest, six regressors modeling residual motion after volume regression, and three regressors modeling baseline, linear, and nonlinear trends for each of two runs. Regressors of interest were convolved with a gamma variate function that modeled a prototypical hemodynamic response before inclusion in the regression model (Cohen, 1997). Idealized signal time courses were timelocked to cue offset (or onset of the anticipatory delay interval) for anticipatory vectors and to feedback onset for outcome regressors.

Statistical maps. The four regressors of interest contrasted: (1) anticipation of responding for monetary gain versus nongain, (2) anticipation of responding to avoid monetary loss versus nonloss, (3) gain versus nongain outcomes, and (4) nonloss versus loss outcomes (equated for the $\sim 66 \%$ trial success probability with regressor 3 ). Anatomical maps of $t$ statistics representing each of these regressors of interest were transformed into $z$ scores, which were spatially normalized by warping to Talairach space, spatially smoothed ( $2 \mathrm{~mm}$ FWHM), and combined into a group map using a meta-analytic formula (average, $Z$ times the square root of $n$ ) (Knutson et al., 2000; Donaldson et al., 2001). To increase statistical power, all gain trial types and loss trial types were collapsed in these contrasts.

For each contrast, an omnibus statistical significance threshold of $p<$ 0.0001 was applied to subcortical and cortical structures that have previously been reported to be recruited in this (Knutson et al., 2000, 2003) and similar (Breiter et al., 2001) incentive paradigms. This threshold approximates a Bonferroni correction for the reduced number of (smoothed) voxels contained by these regions of interest (Knutson et al., 2000). The omnibus threshold for activations in remaining search volumes was set at an order of magnitude higher at $p<0.00001$, uncorrected.

Post hoc analyses I. Signal change in volumes of interest. Differences between groups in event-related signal changes were characterized with VOI analyses, in which time series signal data from the same brain coordinates in both groups were analyzed. We selected three VOI centered a priori on the voxel of maximal event-related activation in a previously published study using this paradigm (Knutson et al., 2003). These VOI consisted of 3 $\mathrm{mm}$ radius spheres centered at the following Talairach coordinates: left $\operatorname{NAcc}(-9,10,0)$, right $\operatorname{NAcc}(11,12,0)$, and $\operatorname{mFC}(1,53,-6)$.

We calculated and analyzed the BOLD signal change following events that were featured in the regression models, such as anticipation of responding to win 5 dollars, or after feedback of gains ("hits"). Signal data were extracted from the time series as follows: (1) signal at each voxel was converted to a (percentage) deviation from the mean for that voxel across the entire time series, (2) signal was averaged by trial type and spatially translated into Talairach space, and (3) signal change values were calculated from the average across the VOI mask at the acquisition observed to be the peak of the hemodynamic response after the event of interest.

For anticipatory activations in the VS, time-lagged signal increases were analyzed in mixed-model ANOVA, with incentive magnitude (four levels: 0,20 cents, 1 dollar, 5 dollars) as within-subject factors and group (two levels: adult and adolescent) as the between-subject factor. For this analysis, nonincentive trials $(n=36)$ were randomly split, with signal from 18 nonincentive trials assigned to the gain analysis and the other 18 trials to the loss analysis. To determine whether VS signal change differed by valence, a second mixed-model ANOVA on the incentive-laden trials was performed, with incentive magnitude (three levels: 20 cents, 1 dollar, 5 dollars) nested within valence (two levels: gain vs loss) as within-subject factors and group (two levels: adult and adolescent) as the betweensubject factor.

To examine differential outcome-elicited signal change in the $\mathrm{mFC}$ VOI, a mixed-model ANOVA was performed, with trial outcome (two levels: hits vs "misses") nested within incentive magnitude (three levels: 20cents, 1 dollar, 5 dollars) nested within valence (two levels: gain vs loss) as within-subject factors and group (two levels: adult and adolescent) as the between-subject factor. In this analysis, we defined hits as notification of obtainment of gains (in gain trials) together with avoidance of losses (in loss trials), and we conversely defined misses as losses (in loss trials) and failures to win gains (in gain trials).

Post hoc analyses II. Group difference maps. We also calculated a statistical map of the differences in activation between adolescents and adults. First, individual subject data were normalized by conversion to percentage-signal change. Second, we performed voxel-wise $t$ tests of the event-related $\beta$ coefficients calculated from the general linear model (using contrasts of the regression model). We restricted our analysis of group activation differences to ventral striatal and mesial frontal brain regions shown to be involved in incentive salience-reward processing in previous reports and only considered voxels that demonstrated suprathreshold (omnibus, $p<0.0001$ ) activation or deactivation for either anticipatory or outcome-based contrast in either group in the present data set. To initially isolate potential regions of voxel-wise differences in each of the four main contrasts, a clustering filter eliminated voxels with no (shared face) nearest-neighbor values above an uncorrected threshold of $|t| \geq 2.077(p<0.05)$ as well as clusters encompassing $<1000 \mu$ l.

To clarify voxels demonstrating a significant group difference in activation, we performed a small-volume correction for multiple comparisons as follows. First, we defined the volume of the (unilateral) VS, including NAcc as $1 \mathrm{cc}$, which corresponds to a sphere with a radius of 6.2 $\mathrm{mm}$. Second, we calculated the adjusted number of comparisons made in each of the right and left VS after isotropic spatial smoothing $(4 \mathrm{~mm}$ FWHM). On the basis of the formula published by Worsley et al. (1992), the number of "resels" encompassed by a $1 \mathrm{cc}$ volume (with a $3.75 \times$ $3.75 \times 2 \mathrm{~mm}$ raw voxel size and $4 \mathrm{~mm}$ FWHM isotropic smoothing) was 12.16 resels per side in the VS. Third, we Bonferroni adjusted the twotailed $t$ test significance value of 0.05 by dividing 0.05 by the number of resels (12.16), resulting in an adjusted threshold of significant group differences of $p<0.0041$.

Behavior analysis. Mixed-model ANOVA of hit rates and reaction times (RT) was performed for different trial types, with incentive valence (two levels: positive and negative) and magnitude (four levels: 0,20 cents, 1 dollar, 5 dollars) as within-subjects factors and group (two levels: adolescent and adult) as the between-subjects factor. To determine whether RT and hit rates (averaged across all trial types) changed over time, a separate ANOVA assessed main effects of time (two levels: run 1 and run 2 ) as the within-subject factor and group (two levels: adolescent and adult) as the between-subject factor. We also analyzed how "happy" and "excited" subjects reported being about seeing the gain cues as well as how "fearful" and "unhappy" subjects were when viewing the loss cues. Mixed-model ANOVA of these four rating series was performed, with incentive magnitude (four levels: 0,20 cents, 1 dollar, 5 dollars) as the within-subjects factor and group (two levels: adolescent and adult) as the between-subject factor.

We also compared head motion between adults and adolescents as a potential between-subjects confound. Output files of compensatory dataset adjustments at each point in the time series (i.e., the I-S, R-L, and A-P displacements required to bring each volume in least-squares alignment with the reference volume) were generated. We then calculated the $\mathrm{SD}$ of the adjustment values at each time point and compared them between adults and adolescents in each of the three directions using independent $t$ tests.

\section{Results}

\section{Behavior and affect}

Adolescents and young adults performed similarly on the MID task. There were no main or interaction effects of valence, magnitude, or age group on rates of successful trials, with an overall hit rate of $69.9 \%$. Within each age group, mean reaction times to targets after the different incentive cues were very similar, differing at most by $\sim 2 \%$. There were no significant main or interaction effects of age group on RT. There was a small main effect of run (time) on slightly reducing reaction time to targets $\left(F_{(1,22)}=\right.$ 6.864; $p=0.0156$ ) from the first task run to the second, but no consequent main effect of run-time on actual hit rates. There was 
no interaction effect of age group times time on either RT or hit rates.

Questionnaire ratings indicated significant main effects of incentive magnitude on each of the four affective ratings, in which participants reported greater happiness $\left(F_{(3,66)}=16.170 ; p<0.0001\right)$ and excitement $\left(F_{(3,66)}=39.673 ; p<0.0001\right)$ as potential gain amounts increased and also reported greater unhappiness $\left(F_{(3,66)}\right.$ $=5.621 ; p=0.0034)$ and fearfulness $\left(F_{(3,66)}=14.949 ; p<0.0001\right)$ as potential loss amounts increased. There were no significant main or interaction effects of age group in any of the four analyses. Comparisons of head motion indicated no significant differences in volumecorrection adjustments between the two groups in all three dimensions.

\section{Statistical maps}

Gain versus nongain anticipation

Anticipation of gain (all amounts collapsed) versus nongain activated the NAcc bilaterally in both adults and adolescents, with more extensive bilateral activation in adults (Fig. 1). The right insula, dorsal midbrain, and dorsal thalamus were also significantly activated in both groups ( $\mathrm{Ta}$ ble 1 ), but this activation was more extensive in adults. Finally, adults but not adolescents showed significant activation of the posterior cingulate.

\section{Loss versus nonloss anticipation}

Anticipation of loss versus nonloss activated only a few regions (at the more inclusive threshold) in either group (Table 1). These included an activation in the right insula in adolescents, deactivation of the $\mathrm{mFC}$ in adults, and activation of the right medial caudate nucleus in both.

\section{Gain versus nongain outcomes}

Activation in response to feedback of gains versus failure to win gains was widespread (Table 2) and was qualitatively similar in the two age groups. In both groups, gain versus nongain outcomes activated several portions of the prefrontal cortex as well as the NAcc and putamen bilaterally (Fig. 1). In addition, portions of the amygdala and hippocampal regions were activated bilaterally by gain outcomes in both groups (Table 2).

\section{Nonloss versus loss outcomes}

Both groups evidenced activation of the left putamen for nonloss versus loss outcomes. Adults uniquely demonstrated activation of the mesial prefrontal cortex and bilateral putamen. A post hoc analysis of signal data indicated that the apparent putamen activations from successful loss avoidance were actually attributable to deactivations after losses. Adolescents also showed a modest activation in the left putamen as well as a small deactivation toward the head of the caudate bilaterally.

\section{VOI analyses}

Anticipatory activations in the VS

As incentive magnitudes increased from 20 cents to 5 dollars, activation (net signal difference from baseline at the acquisition
B.

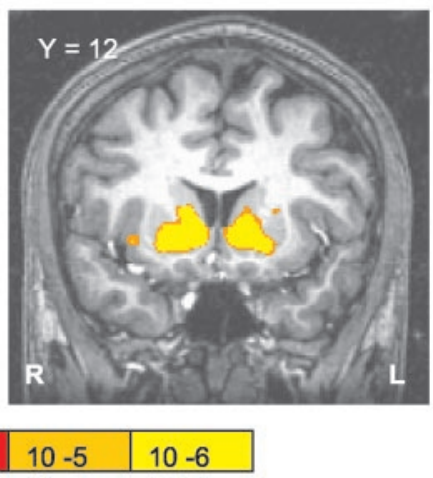

D.

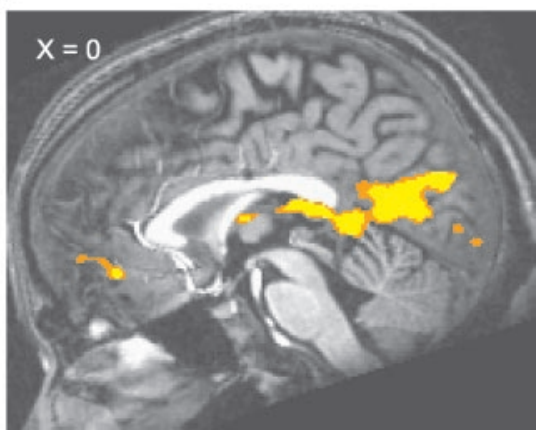

Figure 1. Statistical maps of the gain anticipation and gain outcome contrasts. $A, B$, Statistical maps thresholded at $p<0.0001$ > 3.888) uncorrected illustrate modest activations in ventral striatum anticipating responding for gains (vs nongains) in adolescents $(A)$ but more extensive bilateral activations by this contrast in adults $(B)$. $C, D$, Notification of gains (vs failure to win gains) activated mesial frontal cortex (Brodmann area 10,32) in both adolescents $(C$ ) and adults $(D)$.

of event-related hemodynamic response function peak) increased accordingly in the left and right NAcc (Fig. 2A,B). Specifically, main effects of incentive magnitude were present in the left NAcc for both gain trials $\left(F_{(3,66)}=15.434 ; p<0.0001\right)$ and loss trials $\left(F_{(3,66)}=11.258 ; p<0.0001\right)$. Similar main effects of incentive magnitude were detected in right NAcc for gain trials $\left(F_{(3,66)}=16.100 ; p<0.0001\right)$ and loss trials $\left(F_{(3,66)}=5.819 ; p=\right.$ $0.0017)$. The group by incentive magnitude interaction was significant for gain trials $\left(F_{(3,66)}=4.300 ; p=0.008\right)$ in the right NAcc (but not left Nacc). In the right NAcc, adults demonstrated a larger orderly activation difference as incentive amounts increased from 0 to 5 dollars compared with adolescents (Fig. 2 B), where signal increase from baseline anticipating the large ( 5 dollars) gain was significantly higher in adults than adolescents $(t(22)=2.588 ; p=0.0183)$.

Among incentive trials only, signal increases anticipating potential gains were significantly higher than for potential losses, as indicated by significant main effects of cue valence in left NAcc $\left(F_{(1,22)}=29.634 ; p<0.0001\right)$ and right $\operatorname{NAcc}\left(F_{(1,22)}=11.060\right.$; $p=0.0031)$. There was also a significant magnitude by valence interaction in the left $\operatorname{NAcc}\left(F_{(2,44)}=4.297 ; p=0.020\right)$ but not right NAcc, with magnitude-dependent signal increase in gain trials but not in loss-avoidance trials. There was a trend toward an interaction effect of age group by valence in the right $\operatorname{NAcc}\left(F_{(1,22)}\right.$ $=3.628, p=0.07)$ but not left NAcc. To assess whether some global signal change was responsible for the increase in signal contrast for increasing gains in the VS, time series were similarly calculated and assessed in additional VOIs drawn in cortical gray matter not activated by the task. None of these regions demonstrated any appreciable or orderly increases in net signal change. 
Table 1. Incentive anticipation-elicited activations by subject group

\begin{tabular}{|c|c|c|c|c|c|c|}
\hline \multirow{2}{*}{$\begin{array}{l}\text { Contrast } \\
\text { Gain versus nongain anticipation }\end{array}$} & \multirow[t]{2}{*}{ Region } & \multicolumn{3}{|c|}{ Talairach coordinates } & \multirow[t]{2}{*}{ z-value } & \multirow[t]{2}{*}{ Uncorrected $p$} \\
\hline & & & & & & \\
\hline \multirow[t]{13}{*}{ Adults } & Rinsula & 33 & 17 & 3 & 5.60 & $<0.0001$ \\
\hline & R NACC & 12 & 18 & -3 & 7.044 & $<0.00001$ \\
\hline & L NACC & -12 & 16 & -3 & 7.024 & $<0.00001$ \\
\hline & R medial caudate & 10 & 8 & 1 & 7.274 & $<0.00001$ \\
\hline & L medial caudate & -10 & -1 & 12 & 5.62 & $<0.0001$ \\
\hline & L NACC & -11 & 8 & -1 & 7.476 & $<0.00001$ \\
\hline & R putamen & 21 & 10 & -3 & 6.88 & $<0.00001$ \\
\hline & L midbrain & -4 & -27 & 1 & 5.836 & $<0.00001$ \\
\hline & R midbrain & 8 & -26 & 7 & 5.87 & $<0.0001$ \\
\hline & $\mathrm{R}$ thalamus & 5 & -10 & 12 & 5.950 & $<0.00001$ \\
\hline & $\mathrm{L}$ thalamus & -8 & -21 & 14 & 6.185 & $<0.00001$ \\
\hline & $L$ thalamus & -4 & -22 & 4 & 6.15 & $<0.0001$ \\
\hline & $R$ cuneus & 1 & -75 & 11 & 4.927 & $<0.00001$ \\
\hline \multirow[t]{8}{*}{ Adolescents } & R insula & 39 & 11 & 5 & 4.34 & $<0.0001$ \\
\hline & R NAcc & 11 & 11 & -3 & 3.956 & $<0.0001$ \\
\hline & L NAcc & -7 & 8 & -2 & 5.746 & $<0.00001$ \\
\hline & R extended amygdala & 8 & 4 & -7 & 4.201 & $<0.00001$ \\
\hline & $\mathrm{R}$ dorsal midbrain & 5 & -29 & -1 & 4.634 & $<0.00001$ \\
\hline & L thalamus & -5 & -10 & 10 & 4.14 & $<0.00001$ \\
\hline & L thalamus & -5 & -16 & 10 & 4.177 & $<0.0001$ \\
\hline & L dorsal caudate & -16 & -6 & 17 & 5.094 & $<0.00001$ \\
\hline \multicolumn{7}{|c|}{ Loss versus nonloss anticipation } \\
\hline \multirow[t]{4}{*}{ Adults } & Mesial prefrontal cortex & -5 & 53 & 1 & -5.05 & $<0.0001$ \\
\hline & R parahippocampal gyrus & 27 & 0 & -13 & -3.96 & $<0.0001$ \\
\hline & R mesial caudate & 7 & 10 & 5 & 4.03 & $<0.0001$ \\
\hline & L midbrain & -5 & -27 & -4 & 4.23 & $<0.0001$ \\
\hline \multirow[t]{5}{*}{ Adolescents } & R mesial frontal gyrus & 32 & 47 & 0 & 3.99 & $<0.0001$ \\
\hline & Rinsula & 39 & 17 & 3 & 4.30 & $<0.0001$ \\
\hline & R mesial caudate & 10 & 0 & 14 & 4.35 & $<0.0001$ \\
\hline & R tail caudate & 31 & -39 & 5 & 4.46 & $<0.0001$ \\
\hline & L tail caudate & -30 & -46 & 5 & 4.04 & $<0.0001$ \\
\hline
\end{tabular}

Deactivations presented in italics.

Outcome activations in the $m F C$

There were no significant main effects of incentive amount, group, valence, and outcome on signal change after notifications of incentive trial outcomes in the mFC VOI (Fig. 2C). Consistent with the localization of this VOI (on the basis of activation by the gain versus nongain outcome contrast in a previous data set), a significant outcome by valence interaction $\left(F_{(1,22)}=17.533 ; p=\right.$ 0.0003 ) indicated that in gain trials, but not loss-avoidance trials, there was a difference in signal change between notifications of gains (signal increase) versus failure to win gains (signal decrease). Higher-order interactions with age group and incentive amount were not significant.

\section{Statistical maps of group differences in activation}

To directly compare activation in adolescents and adults, we calculated a statistical difference map for each of the four main event contrasts. On the basis of the criteria described in Materials and Methods, there was only one foci of significant group differences, which was in the gain versus nongain anticipation contrast. $t$ test maps indicated reduced gain anticipation activation in the right VS in adolescents compared with adults (Fig. 3), which was centered at Talairach coordinates $(9,17,-2)$, where the groups differed in activation at $t=3.334, p=0.003$ (corrected $p<0.05$ ). Peripheral voxels demonstrating group differences at an uncorrected $p<0.05$ extended anteriorly and laterally into the right NAcc and right anterior insula and extended posteriorly to the right amygdala.

\section{Relationship between gain anticipation activation, age, and positive affect}

Because the post hoc analyses collectively indicated an age groupbased difference in right NAcc activation by anticipation of high gains, we sought to determine how age related to activation more directly in this area. In addition, we also attempted to replicate data from a previous study using the MID task in healthy adults (Knutson et al., 2001a), in which signal elevation in the right anterior VS during anticipation of responding for gains correlated with self-reported positive affective reactions to presentation of the large (i.e., 5 dollars) gain cue. Age and self-reports of happy and excited when seeing the highest reward cue were correlated with net signal increases during anticipation of potential gain. As in the previous report, each affect rating was calculated as the net difference from the mean rating number the subject endorsed for the adjective for all other cues (to control for response biases). Across both groups, neither mean-deviated happy nor excited ratings about the 5 dollar gain cue correlated with age (both $|r|<0.06)$.

Age $(r=0.437 ; p=0.033)$ and net ratings of excited about the 5 dollar gain $(r=0.437 ; p=0.033)$ showed similar positive correlations with activation during anticipation of the 5 dollar gain in the right NAcc VOI. The positive correlation with ratings of happy did not reach significance. Neither rating adjective nor age correlated singly with net anticipatory signal contrast in the left NAcc. To clarify independent effects of each of the age and affect on VS gain anticipation activation, we performed a multi- 
Table 2. "Hit" versus "miss" outcome feedback-elicited activations by subject group

\begin{tabular}{|c|c|c|c|c|c|c|}
\hline \multirow{2}{*}{$\begin{array}{l}\text { Contrast } \\
\text { Gain versus nongain outcome }\end{array}$} & \multirow[t]{2}{*}{ Region } & \multicolumn{3}{|c|}{ Talairach coordinates: } & \multirow[t]{2}{*}{ z-value } & \multirow[t]{2}{*}{ Uncorrected $p$} \\
\hline & & & & & & \\
\hline \multirow[t]{16}{*}{ Adults } & R mesial PFC & 12 & 45 & -11 & 6.012 & $<0.00001$ \\
\hline & L mesial PFC & -1 & 41 & -11 & 5.334 & $<0.00001$ \\
\hline & RIFG & 28 & 28 & -13 & 5.23 & $<0.0001$ \\
\hline & LIFG & -29 & 29 & -11 & 4.13 & $<0.0001$ \\
\hline & R NAcC & 7 & 15 & 0 & 5.24 & $<0.0001$ \\
\hline & L NACC & -9 & 15 & 0 & 4.82 & $<0.0001$ \\
\hline & R NAcc & 11 & 10 & -5 & 5.329 & $<0.00001$ \\
\hline & L putamen & -22 & 6 & -4 & 4.93 & $<0.0001$ \\
\hline & L putamen & -28 & -12 & -1 & 5.57 & $<0.0001$ \\
\hline & Ramygdala & 21 & -1 & -11 & 6.184 & $<0.00001$ \\
\hline & L amygdala & -17 & -3 & -11 & 5.008 & $<0.00001$ \\
\hline & thalamus & 1 & -5 & 10 & 5.15 & $<0.0001$ \\
\hline & R hippocampus & 23 & -22 & -5 & 5.755 & $<0.00001$ \\
\hline & L hippocampus & -17 & -27 & -3 & 5.129 & $<0.00001$ \\
\hline & R posterior cingulate & 14 & -56 & 20 & 6.35 & $<0.0001$ \\
\hline & Mesial posterior cingulate & 2 & -61 & 17 & 7.04 & $<0.0001$ \\
\hline \multirow[t]{17}{*}{ Adolescents } & Mesial PFC & 1 & 46 & -4 & 5.609 & $<0.00001$ \\
\hline & Mesial PFC & -4 & 40 & -4 & 5.554 & $<0.00001$ \\
\hline & RIFG & 42 & 29 & 10 & 4.89 & $<0.0001$ \\
\hline & LIFG & -37 & 29 & 10 & 4.88 & $<0.0001$ \\
\hline & R MFG & 29 & 33 & -15 & 4.66 & $<0.0001$ \\
\hline & R NACC & 11 & 17 & -3 & 5.315 & $<0.00001$ \\
\hline & L NACC & -7 & 16 & -1 & 5.444 & $<0.00001$ \\
\hline & R putamen & 27 & -8 & 8 & 5.98 & $<0.0001$ \\
\hline & L putamen & -21 & -10 & 10 & 4.98 & $<0.0001$ \\
\hline & RSLEA & 11 & 4 & -9 & 5.205 & $<0.00001$ \\
\hline & LSLEA & -14 & 2 & -9 & 4.981 & $<0.00001$ \\
\hline & R Phip gyrus & -23 & -29 & -6 & 4.75 & $<0.0001$ \\
\hline & L Phip gyrus & 22 & -30 & -2 & 4.96 & $<0.0001$ \\
\hline & R Dorsal Caudate & 19 & -1 & 16 & 4.50 & $<0.0001$ \\
\hline & L dorsal caudate & -8 & -4 & 16 & 4.57 & $<0.0001$ \\
\hline & L dorsal thalamus & -4 & -20 & 17 & 5.464 & $<0.00001$ \\
\hline & L posterior cingulate & -4 & -52 & 18 & 4.12 & $<0.0001$ \\
\hline \multicolumn{7}{|c|}{ Nonloss versus loss outcome } \\
\hline \multirow[t]{6}{*}{ Adults } & R putamen & 24 & 5 & -2 & 5.800 & $<0.00001$ \\
\hline & Lputamen & -23 & -1 & 3 & 5.758 & $<0.00001$ \\
\hline & R mesial PFC & 7 & 46 & -12 & 4.741 & $<0.00001$ \\
\hline & L mesial PFC & -2 & 46 & -13 & 4.64 & $<0.00001$ \\
\hline & R posterior cingulate & 1 & -50 & 24 & 4.22 & $<0.0001$ \\
\hline & R premotor cortex & 52 & -3 & 5 & 5.212 & $<0.00001$ \\
\hline \multirow[t]{4}{*}{ Adolescents } & $R$ medial caudate & 8 & 6 & -2 & -4.249 & $<0.0001$ \\
\hline & L medial caudate & -6 & 6 & 4 & -4.544 & $<0.00001$ \\
\hline & L putamen & -26 & -14 & 2 & 4.466 & $<0.00001$ \\
\hline & Rsuperior midbrain & 7 & -28 & 1 & -4.443 & $<0.00001$ \\
\hline
\end{tabular}

Deactivations presented in italics.

ple regression analysis for each of the right NAcc and left NAcc VOI, in which age and the net excited rating about the 5 dollar gain were simultaneously entered as independent variables, and 5 dollar gain anticipation activation was the dependent variable. This resulted in slightly higher and similar partial correlations between activation and each of self-reported excitement $(\beta=$ $0.453 ; p=0.014)$ and age $(\beta=0.453 ; p=0.014)$ in the right NAcc (Fig. 4). In addition, self-reported excitement also correlated with gain anticipation in the left NAcc $(\beta=0.502 ; p=$ 0.020 ) when age was controlled for, but age did not independently correlate with gain anticipation.

\section{Discussion}

These data collectively indicate similar patterns of brain responses to incentive cues and outcomes in adolescents and adults. However, direct group comparison revealed an adolescent decrement in gain anticipation activation in the right VS, cen- tered in the NAcc at coordinates near previously published (Knutson et al., 2001a, 2003) peak activation foci for the gain versus nongain anticipation contrast. Moreover, age directly correlated with high-gain anticipation across all subjects in the right Nacc, independent of individual differences in positive affect about the prospect of winning large gains. In both groups, there was bilateral activation of the VS during anticipation of gains, as well as selective activation of the mFC by notification of gains (contrasted with notification of missed gains). When individual differences in age were controlled, signal increase during anticipation of the high ( 5 dollars) gain in both the right and left NAcc correlated with self-reported excitement about the opportunity to respond for that gain.

Anticipation of responding to avoid loss also elicited a modest activation in the right mesial caudate in both groups. VOI analysis indicated that although the left and right NAcc were prefer- 
A.

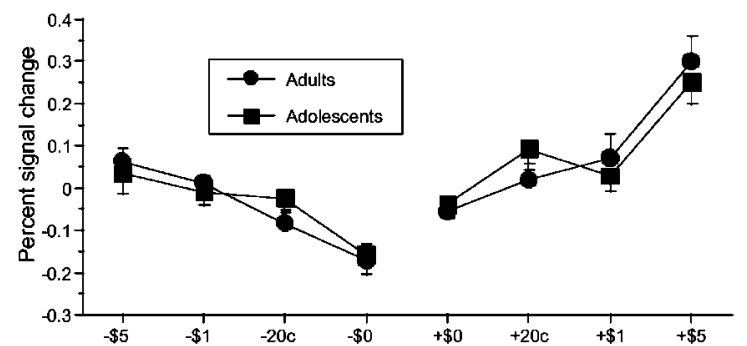

B.

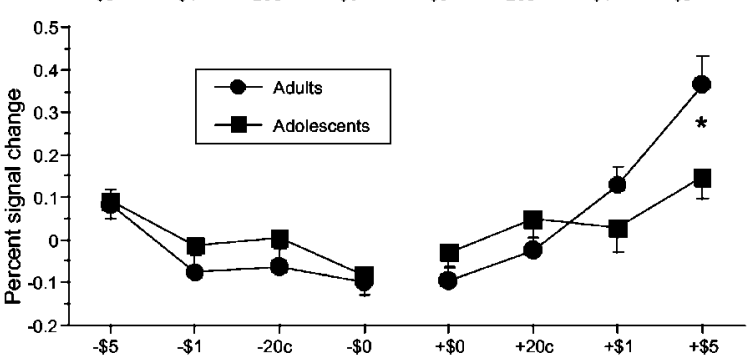

C.

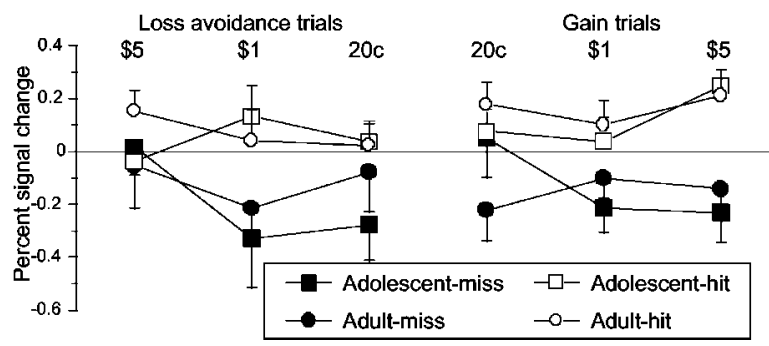

Figure 2. Event-elicited signal change in V0l. Each VOI was a sphere $6 \mathrm{~mm}$ in diameter centered on the Talairach coordinates showing the highest event-related $z$ statistic in a previous experiment using this paradigm (Knutson et al., 2003). These were the gain versus nongain anticipation contrast maxima in the left $\operatorname{NAcc}(-9,10,0 ; A)$ and right $\operatorname{NAcc}(11,12,0 ; B)$ and the gain versus nongain outcome maxima in the $\mathrm{mFC}(1,53,-6 ; C)$. Data were averaged across the VOl and extracted during the acquisition corresponding to the lagged peak of the event-related hemodynamic response in adolescents (squares) and adults (circles) and are expressed as percentage signal change from baseline, where baseline is mean signal value averaged across the entire time series. There was a main effect of incentive amount (from 0 to 5 dollars) on activation during anticipation of both gains and losses in the left and right NAcc, where a main effect of valence among the incentive-laden trials indicated greater anticipatory activation by potential gains compared with losses in both VOl. There was a significant age group times incentive amount interaction effect on anticipatory activation in the gain trial series in the right NAcc, with significantly $\left({ }^{*} p<0.05\right)$ lower signal increase while anticipating responding to win the large (5 dollars) gains in adolescents. In the $\mathrm{mFC} \mathrm{VOl,} \mathrm{a} \mathrm{significant} \mathrm{valence} \mathrm{by} \mathrm{outcome} \mathrm{interac-}$ tion indicated that in gain trials (but not in loss-avoidance trials), notification of "hits" significantly increased signal relative to notification of "misses." There were no significant main or interactive effects of age group on activation in the $\mathrm{mFC} \mathrm{VOI.}$

entially recruited during anticipation of responding for gains compared with responding to avoid losses, anticipatory activation during loss-avoidance trials also increased as the amount of potential loss increased. Money loss notification revealed putamen deactivation in both groups, which was extensive and bilateral in the adults but modest in adolescents. These deactivations raise the possibility that ventral putamen deactivations result not only from omission of expected reward (McClure et al., 2003; O'Doherty et al., 2003) but also from failures to preserve reward.

These data extend to adolescents several previous findings in adults, notably magnitude-sensitive activation of the VS by anticipation of responding to win money (Knutson et al., 2001a). The correlation between positive affect about winning large gains and actual signal increase in the VS during high-gain anticipation, first reported by Knutson et al. (2001a), underscores the recruitment of the VS by gain-incentive salience in both adoles-

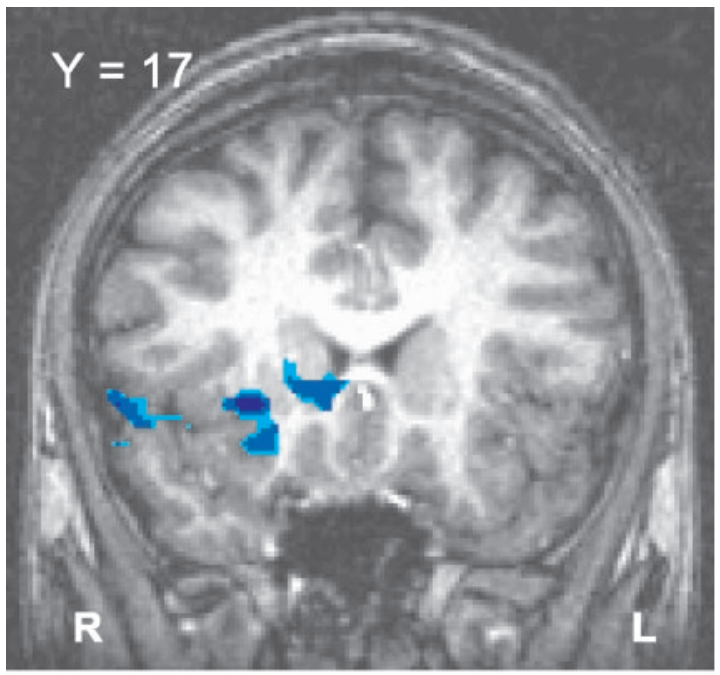

$P<.05$ $\mathrm{P}<.01$

Figure 3. Age difference in gain anticipation activation. Voxel-wise $t$ tests of age group differences in event-related regression coefficients were performed in regions of interest. $\mathrm{Ad}$ olescents showed decreased gain anticipation activation compared with adults in the right ventral striatum. For illustrative purposes, maps were thresholded at $p<0.05$ with exclusion of noncontiguous voxels and clusters $<1000 \mu \mathrm{l}$. The group difference map depicts this activation deficit in adolescents, where voxel-wise group differences are graded on the basis of uncorrected $p$ values, and the groups differ at a Bonferroni-corrected $p<0.05$ in voxels centered at 9,17 , and -2 .

cents and adults. As with adults in this and previous (Knutson et al., 2001b, 2003) datasets, notification of gains also activated the $\mathrm{mFC}$ in adolescents. We note that in this outcome-based contrast, the gain trials were bifurcated. If the activation in $\mathrm{mFC}$ was a delayed hemodynamic response to anticipation of gains, anticipatory activations preceding losses and preceding gains would be similar and would have negated this contrast. Here, as in a recent report (Knutson et al., 2003), gain notification generally resulted in signal increases over baseline in the $\mathrm{mFC}$, whereas failures to win resulted in decreases from baseline.

We suggest that the age differences in right NAcc gain anticipation activation may have focal neurodevelopmental underpinnings. Although reports on normative laterality of subcortical brain structures are contradictory, and to our knowledge there are no reports of differential rates of development of the right versus left striatum from early adolescence to young adulthood, we note that caudate volume decreases by $\sim 8 \%$ from $12-19$ years of age (Castellanos et al., 2002), with the caudate head having the largest proportional reduction across childhood into adolescence (Thompson et al., 2000). Sowell et al. (1999) illustrated significantly decreased ventral striatal volume in adults $23-30$ years of age compared with adolescents $12-16$ years of age in mesial caudate areas, where, in this study, suprathreshold activation was detected in adults but not in adolescents.

Alternatively, the right-selective deficit in adolescent recruitment of the VS may have reflected developmental deficits in attentional control while performing the MID task. Right caudate head size and attention deficit symptomatology have been positively correlated both in children with attention deficit-hyperactivity disorder and controls (Mataro et al., 1997). Here, the posterior cingulate, cuneus, and portions of visual cortex were activated by gain feedback more extensively in adults, possibly reflecting greater attention and processing of motivationally salient stimuli (Bradley et al., 2003). 
A.

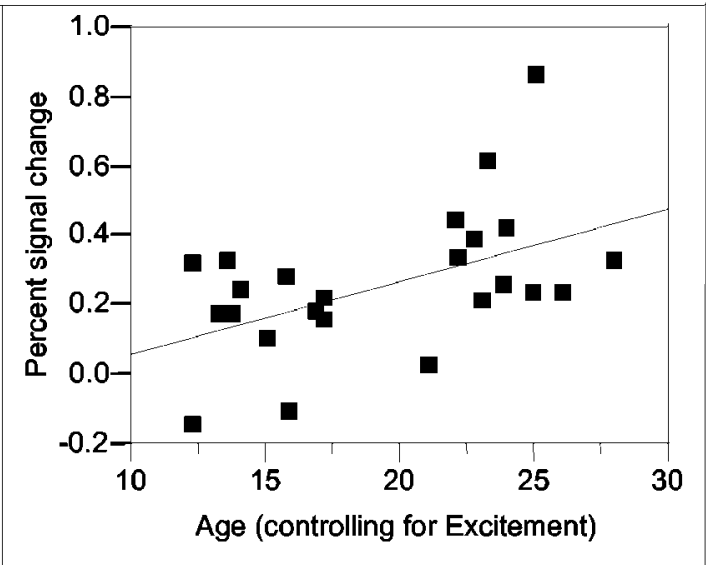

B.

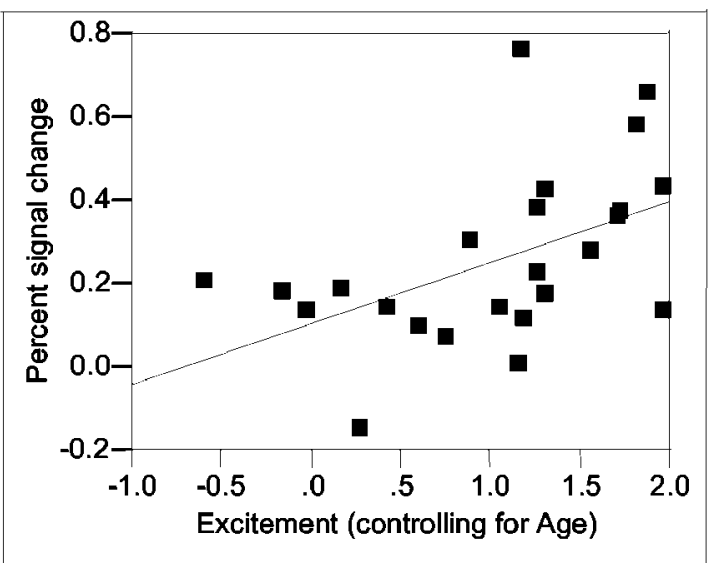

Figure 4. Affective ratings and age independently correlate with gain-anticipatory activation in the right nucleus accumbens. Using multiple regression, activation in the right nucleus accumbens while subjects anticipated the opportunity to respond for large (5 dollars) gains was independently correlated with age and mean-deviated excitement ratings when seeing anticipatory cues signaling the availability to respond for the large gains. There were nearly identical independent correlations between anticipatory activation and the subject's age (while controlling for self-reported excitement; $\beta=0.452 ; p=0.014 ; A$ ) as well as between anticipatory activation and self-reported excitement (while controlling for age; $\beta=0.452 ; p=0.014 ; B$ ). These partial correlations are graphically represented here as leverage plots.

Our results do not support the hypothesis that adolescents have increased reward cue-elicited anticipatory VS activation. This finding provides more support for an adolescent VS activation deficit rather than a VS activation surfeit account of adolescent risky behavior, in which adolescents may seek more extreme incentives as a way of compensating for low VS activity levels (Spear, 2000). These data are also consistent with findings from psychosocial research that adolescents are less optimistically biased about obtaining future rewards compared with adults (Millstein, 1993). However, we note that the MID task has an effortful component, and differential behavioral effects of NAcc dopamine (DA) depletion on operant schedules of reinforcement suggest that the NAcc is selectively engaged when mobilization of an operant response for gain is required (Salamone et al., 2003). The adolescent deficit may not exist or may even be reversed in a nonoperant (Pavlovian) paradigm, inasmuch as gain outcome (consummatory) activations were similar between the two age groups. Future research with a similar paradigm that modulates work requirement could address this systematically.
These differences may also reflect neurochemical development of the NAcc from adolescence to adulthood. Although FMRI does not measure neurochemical activity, our argument implicitly assumes that activation in the VS may be related to changes in neuronal membrane excitability in that region (Logothetis, 2003). DA is a potent modulator of postsynaptic membrane excitability in the NAcc, where administration of DA agonists increases activation in VS and forebrain regions in rats (Chen et al., 1997). Thus, endogenous DA release may partially account for NAcc activation while anticipating responses for reward (Phillips et al., 2003). Developmental research has revealed numerous changes in the dopaminergic system between adolescence and adulthood in rats (Andersen and Gazzara, 1993; Teicher et al., 1993), nonhuman primates (Fairbanks et al., 1999), and humans (Seeman et al., 1987; Meng et al., 1999; Haycock et al., 2003).

The monetary incentive delay task has many strengths, including the ability to dissociate trial components (i.e., gain anticipation vs outcome) in time. Here, the dissociation highlighted a quantitative difference in gain anticipatory (but not outcomeelicited) VS activation in adolescents versus adults. However, the MID task also has several limitations. First, this task does not evoke activation associated with anticipation of losses as powerfully as activation associated with anticipation of gains, which makes comparison of loss anticipatory activation across groups difficult. Second, subjects must make a rapid behavioral response in different trials. Although we hypothesized that right VS activation might index a high-arousal positive-affective state, the observed activations might be associated with a combination of this affective state and preparation of movements that lead to reward acquisition (although a purely movement-based account is insufficient to explain right VS activation, because exactly the same movements are required for escaping losses as for acquiring gains).

Additionally, although adolescents and adults showed remarkably similar patterns of brain activation in terms of spatial localization, comparison of these groups raises nontrivial methodological issues. For instance, although the brain reaches maximum volume by approximately 14 years of age (Courchesne et al., 2000), the structure of adolescent brains differs slightly from those of adults (Giedd et al., 1999), and the resolution of the structural scans acquired in this experiment was not sufficient to enable structure to be used as a covariate in these analyses. Individual differences in the rate of development might also result in variable functional patterns of activation in adolescents (for review, see Casey et al., 2000), which could reduce group activation maps. Unfortunately, we did not control for precise pubertal development using any standardized measures. Moreover, the sample size of this experiment precluded systematic investigation of gender differences in the development of incentive circuitry. Finally, although the focused slice montage used in this experiment allowed exquisite localization of patterns of activation in the VS, the speed of data acquisition precluded gathering data from more superior regions of the cerebral cortex that might play roles in movement and attention (Knutson et al., 2003).

In conclusion, these data indicate qualitative similarities overall in the brain regions recruited by incentive processing in healthy adolescents and adults. However, the data reveal reduced right VS activation by gain anticipation in adolescents, which appears to result from a reduced discrimination of activationresponses between nonincentive trials and high gain trials compared with adults (Fig. $2 B$ ). Understanding developmental dif- 
ferences in incentive processing may provide important clues for understanding risky behaviors in youth. Some theorists have postulated that hypofunctioning dopaminergic mesolimbic incentive circuitry underlies risky adolescent behavior (Blum et al., 2000) as well as vulnerability for development of substance use disorders (Estroff et al., 1989; Clark et al., 1998). To determine whether individual differences in this circuitry relate to problem behavior, future imaging research can incorporate prospective measurement of risk taking as well as impulsivity, such as the Iowa card-playing task (Bechara and Damasio, 2002) and calculating the rate of devaluation of reward with delay to presentation (Petry and Casarella, 1999). Finally, additional research on incentive processing in at-risk adolescent populations is of interest to determine whether these youth might show pronounced deficits in motivational circuitry.

\section{References}

Achenbach TM (1991) Manual for the child behavior checklist/4-18 and 1991 profile. Burlington, VT: University of Vermont.

Andersen SL, Gazzara RA (1993) The ontogeny of apomorphine-induced alterations of neostriatal dopamine release: effects on spontaneous release. J Neurochem 61:2247-2255.

Arnett J (1992) Reckless behavior in adolescence: a developmental perspective. Dev Rev 12:391-409.

Bechara A, Damasio H (2002) Decision-making and addiction (part I): impaired activation of somatic states in substance dependent individuals when pondering decisions with negative future consequences. Neuropsychologia 40:1675-1689.

Blum K, Braverman ER, Holder JM, Lubar JF, Monastra VJ, Miller D, Lubar JO, Chen TJ, Comings DE (2000) Reward deficiency syndrome: a biogenetic model for the diagnosis and treatment of impulsive, addictive, and compulsive behaviors. J Psychoactive Drugs 32[Suppl i-iv]:1-112.

Bradley MM, Sabatinelli D, Lang PJ, Fitzsimmons JR, King W, Desai P (2003) Activation of the visual cortex in motivated attention. Behav Neurosci 117:369-380.

Breiter HC, Aharon I, Kahneman D, Dale A, Shizgal P (2001) Functional imaging of neural responses to expectancy and experience of monetary gains and losses. Neuron 30:619-639.

Cardinal RN, Pennicott DR, Sugathapala CL, Robbins TW, Everitt BJ (2001) Impulsive choice induced in rats by lesions of the nucleus accumbens core. Science 292:2499-2501.

Casey BJ, Giedd JN, Thomas KM (2000) Structural and functional brain development and its relation to cognitive development. Biol Psychol 54:241-257.

Castellanos FX, Lee PP, Sharp W, Jeffries NO, Greenstein DK, Clasen LS, Blumenthal JD, James RS, Ebens CL, Walter JM, Zijdenbos A, Evans AC, Giedd JN, Rapoport JL (2002) Developmental trajectories of brain volume abnormalities in children and adolescents with attention-deficit/ hyperactivity disorder. JAMA 288:1740-1748.

Chambers RA, Taylor JR, Potenza MN (2003) Developmental neurocircuitry of motivation in adolescence: a critical period of addiction vulnerability. Am J Psychiatry 160:1041-1052.

Chen YC, Galpern WR, Brownell AL, Matthews RT, Bogdanov M, Isacson O, Keltner JR, Beal MF, Rosen BR, Jenkins BG (1997) Detection of dopaminergic neurotransmitter activity using pharmacologic MRI: correlation with PET, microdialysis, and behavioral data. Magn Reson Med 38:389-398.

Clark DB, Kirisci L, Tarter RE (1998) Adolescent versus adult onset and the development of substance use disorders in males. Drug Alcohol Depend 49:115-121.

Cohen MS (1997) Parametric analysis of fMRI data using linear systems methods. NeuroImage 6:93-103.

Courchesne E, Chisum HJ, Townsend J, Cowles A, Covington J, Egaas B, Harwood M, Hinds S, Press GA (2000) Normal brain development and aging: quantitative analysis at in vivo MR imaging in healthy volunteers. Radiology 216:672-682.

Cox RW (1996) AFNI: software for analysis and visualization of functional magnetic resonance neuroimages. Comput Biomed Res 29:162-173.

Donaldson DI, Petersen SE, Ollinger JM, Buckner RL (2001) Dissociating state and item components of recognition memory using fMRI. NeuroImage 13:129-142.

Elliott R, Dolan RJ, Frith CD (2000) Dissociable functions in the medial and lateral orbitofrontal cortex: evidence from human neuroimaging studies. Cereb Cortex 10:308-317.

Estroff TW, Schwartz RH, Hoffmann NG (1989) Adolescent cocaine abuse. Addictive potential, behavioral and psychiatric effects. Clin Pediatr (Phila) 28:550-555.

Fairbanks LA, Fontenot MB, Phillips-Conroy JE, Jolly CJ, Kaplan JR, Mann JJ (1999) CSF monoamines, age and impulsivity in wild grivet monkeys (Cercopithecus aethiops aethiops). Brain Behav Evol 53:305-312.

Furby L, Beyth-Marom R (1992) Risk taking in adolescence: a decisionmaking perspective. Developmental Rev 12:1-44.

Gaillard WD, Grandin CB, Xu B (2001) Developmental aspects of pediatric fMRI: considerations for image acquisition, analysis, and interpretation. NeuroImage 13:239-249.

Gardner W, Herman J (1991) Adolescents' AIDS risk taking: a rational choice perspective. In: Adolescents in the AIDS epidemic (Gardner W, Millstein S, Wilcox B, eds), pp 17-34. San Francisco: Jossey-Bass.

Giedd JN, Blumenthal J, Jeffries NO, Castellanos FX, Liu H, Zijdenbos A, Paus T, Evans AC, Rapoport JL (1999) Brain development during childhood and adolescence: a longitudinal MRI study. Nat Neurosci 2:861-863.

Haycock JW, Becker L, Ang L, Furukawa Y, Hornykiewicz O, Kish SJ (2003) Marked disparity between age-related changes in dopamine and other presynaptic dopaminergic markers in human striatum. J Neurochem 87:574-585.

Knutson B, Westdorp A, Kaiser E, Hommer D (2000) FMRI visualization of brain activity during a monetary incentive delay task. NeuroImage 12:20-27.

Knutson B, Adams CM, Fong GW, Hommer D (2001a) Anticipation of increasing monetary reward selectively recruits nucleus accumbens. J Neurosci 21:RC159.

Knutson B, Fong GW, Adams CM, Varner JL, Hommer D (2001b) Dissociation of reward anticipation and outcome with event-related fMRI. NeuroReport 12:3683-3687.

Knutson B, Fong GW, Bennett SM, Adams CM, Hommer D (2003) A region of mesial prefrontal cortex tracks monetarily rewarding outcomes: characterization with rapid event-related fMRI. NeuroImage 18:263-272.

Logothetis NK (2003) The underpinnings of the BOLD functional magnetic resonance imaging signal. J Neurosci 23:3963-3971.

Mataro M, Garcia-Sanchez C, Junque C, Estevez-Gonzalez A, Pujol J (1997) Magnetic resonance imaging measurement of the caudate nucleus in adolescents with attention-deficit hyperactivity disorder and its relationship with neuropsychological and behavioral measures. Arch Neurol 54:963-968.

McClure SM, Berns GS, Montague PR (2003) Temporal prediction errors in a passive learning task activate human striatum. Neuron 38:339-346.

Meng SZ, Ozawa Y, Itoh M, Takashima S (1999) Developmental and agerelated changes of dopamine transporter, and dopamine D1 and D2 receptors in human basal ganglia. Brain Res 843:136-144.

Millstein FG (1993) Perceptual, attributional, and affective processes in perceptions of vulnerability through the life span. In: Adolescent risk taking (Bell NJ, Bell RW, eds), pp 55-65. Newbury Park, CA: Sage Publications.

Neter J, Kutner MH, Machtsheim CJ, Wasserman W (1996) Applied linear statistical models, Ed 4. Chicago: Irwin.

O’Doherty J, Critchley H, Deichmann R, Dolan RJ (2003) Dissociating valence of outcome from behavioral control in human orbital and ventral prefrontal cortices. J Neurosci 23:7931-7939.

Petry NM, Casarella T (1999) Excessive discounting of delayed rewards in substance abusers with gambling problems. Drug Alcohol Depend 56:25-32.

Phillips PE, Stuber GD, Heien ML, Wightman RM, Carelli RM (2003) Subsecond dopamine release promotes cocaine seeking. Nature 422:614-618.

Reppucci ND (1999) Adolescent development and juvenile justice. Am J Community Psychol 27:307-326.

Salamone JD, Correa M (2002) Motivational views of reinforcement: implications for understanding the behavioral functions of nucleus accumbens dopamine. Behav Brain Res 137:3-25.

Salamone JD, Correa M, Mingote S, Weber SM (2003) Nucleus accumbens dopamine and the regulation of effort in food-seeking behavior: implica- 
tions for studies of natural motivation, psychiatry, and drug abuse. J Pharmacol Exp Ther 305:1-8.

Seeman P, Bzowej NH, Guan HC, Bergeron C, Becker LE, Reynolds GP, Bird ED, Riederer P, Jellinger K, Watanabe S, Tourtellotte WW (1987) Human brain dopamine receptors in children and aging adults. Synapse 1:399-404.

Sowell ER, Thompson PM, Holmes CJ, Jernigan TL, Toga AW (1999) In vivo evidence for post-adolescent brain maturation in frontal and striatal regions. Nat Neurosci 2:859-861.

Spear LP (2000) The adolescent brain and age-related behavioral manifestations. Neurosci Biobehav Rev 24:417-463.

Tangney JP, Hill-Barlow D, Wagner PE, Marschall DE, Borenstein JK, Sanftner J, Mohr T, Gramzow R (1996) Assessing individual differences in constructive versus destructive responses to anger across the lifespan. J Pers Soc Psychol 70:780-796.

Teicher MH, Barber NI, Gelbard HA, Gallitano AL, Campbell A, Marsh E, Baldessarini RJ (1993) Developmental differences in acute nigrostriatal and mesocorticolimbic system response to haloperidol. Neuropsychopharmacology 9:147-156.

Thompson PM, Giedd JN, Woods RP, MacDonald D, Evans AC, Toga AW (2000) Growth patterns in the developing brain detected by using continuum mechanical tensor maps. Nature 404:190-193.

Worsley KJ, Evans AC, Marrett S, Neelin P (1992) A three-dimensional statistical analysis for CBF activation studies in human brain. J Cereb Blood Flow Metab 12:900-918. 